\title{
Stochastic Dynamics of Membrane Protrusion Mediated by the DOCK180/Rac Pathway in Migrating Cells
}

\author{
Erik S. Welf and Jason M. Haugh \\ Department of Chemical and Biomolecular Engineering, North Carolina State University, Box 7905, \\ Raleigh, NC 27695, USA
}

\begin{abstract}
Cell migration is regulated by processes that control adhesion to extracellular matrix (ECM) and force generation. While our fundamental understanding of how these control mechanisms are actuated at the molecular level (signal transduction) has been refined over many years, appreciation of their dynamics has grown more recently. Here, we formulate and analyze by stochastic simulation a quantitative model of signaling mediated by the integrin family of adhesion receptors. Nascent adhesions foster the activation of the small GTPase Rac by at least two distinct signaling pathways, one of which involves tyrosine phosphorylation of the scaffold protein paxillin and formation of multiprotein complexes containing the guanine nucleotide exchange factor DOCK180. Active Rac promotes protrusion of the cell's leading edge, which in turn enhances the rate of nascent adhesion nucleation; we call this feedback mechanism the core protrusion cycle. Protrusion is antagonized by stable adhesions, which form by myosin-dependent maturation of nascent adhesions, and we propose here a feedforward mechanism mediated by the tyrosine kinase c-Src by which this antagonism is regulated so as to allow transient protrusion at higher densities of ECM. We show that this "buffering of inhibition" mechanism, coupled with the core protrusion cycle, is capable of tuning the frequencies of protrusion and adhesion maturation events.
\end{abstract}

\section{Keywords}

Integrins; Focal adhesions; Signal transduction; Paxillin; Src-family kinases; Myosin

\section{INTRODUCTION}

Cell motility and migration is a fascinating and complex process that requires coordinated actuation and regulation of membrane protrusion and adhesion processes. Given the essential roles of cell migration in various aspects of human health, such as wound repair, blood vessel formation, development, and the immune response, it is difficult to overstate the importance of understanding its underlying biochemical and mechanical control mechanisms in greater and more quantitative detail.

Dynamic protrusion of the cell front is driven by actin polymerization in the lamellipodium via the activity of the small GTPase Rac. ${ }^{13,25,29}$ Protrusion promotes formation of adhesive

(C) 2010 Biomedical Engineering Society

Address correspondence to Jason M. Haugh, Department of Chemical and Biomolecular Engineering, North Carolina State University, Box 7905, Raleigh, NC 27695, USA. jason_haugh@ncsu.edu.

ELECTRONIC SUPPLEMENTARY MATERIAL

The online version of this article (doi:10.1007/s12195-010-0100-8) contains supplementary material, which is available to authorized users. 
contacts through binding of receptors called integrins to the insoluble extracellular matrix (ECM), followed by clustering of the integrins and recruitment of various cytoplasmic and membrane proteins to the resulting complexes. ${ }^{10,17}$ Thus, these adhesions simultaneously anchor cells to the ECM and provide a scaffold for biochemical signaling events. ${ }^{26,41}$ Recent studies suggest that different types of adhesions play distinct roles during cellular protrusion. Smaller, nascent adhesions are relatively short-lived structures; they assemble in regions of active actin polymerization at the cell's leading edge and are turned over at the lamellipodiumlamellum interface, coinciding with faster rates of actin depolymerization in the lamellum.6, ${ }^{10} \mathrm{~A}$ small fraction of the nascent adhesions mature to form larger, more dynamically stable adhesions, coinciding with a pause or halt of protrusion in their vicinity. ${ }^{6,40}$ The maturation process responds, by a mechanism that is not yet completely understood, to contractile forces exerted by actomyosin.

Nascent adhesions mediate activation of Rac through at least two pathways involving distinct guanine nucleotide exchange factor (GEF) activities. One of the pathways is a seemingly linear sequence involving focal adhesion kinase (FAK)-dependent phosphorylation of the adaptor protein paxillin on $\mathrm{Tyr}^{31}$ and $\mathrm{Tyr}^{118}$ and recruitment of the CrkII adaptor and the unconventional Rac-GEF DOCK180. ${ }^{5,15,22,28,38}$ Phosphorylation of the Tyr ${ }^{31}$ and Tyr ${ }^{118}$ sites on paxillin facilitate numerous migration-related signaling events, and expression of either paxillin with those sites removed (Y31F/Y118F ${ }^{23}$ or a dominant-negative DOCK180 ${ }^{34}$ severely impairs cell migration. Another Rac activation pathway involves a positive feedback loop whereby paxillin phosphorylated on $\operatorname{Ser}^{273}$ mediates recruitment of the scaffold protein GIT1, which in turn coordinates binding of $\beta$ PIX, a Rac-GEF, and PAK, a Rac effector kinase; PAK phosphorylates paxillin on $\operatorname{Ser}^{273}$ (among other substrates), completing the feedback circuit. ${ }^{20}$ The GIT1/ $\beta$ PIX/PAK pathway is required for activation of Rac, protrusion, spreading and migration ${ }^{14,16,31,37,39}$ and plays a role in adhesion dynamics. ${ }^{42}$ The relative importance of the two Rac activation pathways, especially across different cellular/microenvironmental contexts, remains to be systematically characterized.

Computational modeling has emerged as a useful tool for understanding and synthesizing hypotheses about how adhesive and cytoskeletal dynamics generate diverse cell migration behaviors. ${ }^{18}$ Numerous studies have used this approach to examine the mechanical aspects of cell adhesion and migration, ${ }^{4,8,21}$ yet relatively few models have focused on the regulation of cell motility by signaling pathways, as the interface between the underlying mechanics and chemistry is not well understood. ${ }^{36}$ Through computational modeling, we have shown that the aforementioned $\beta \mathrm{PIX} / \mathrm{Rac} / \mathrm{PAK}$ feedback loop is capable of evoking transiently amplified protrusion behavior, which is opposed by feedback loops that promote maturation of stable adhesions. ${ }^{7}$ The protrusion/adhesion phenotypes are reminiscent of experimental observations in CHO.K1 cells; consistent with model predictions, leading edge protrusion is optimized at intermediate ECM density, which balances a high rate of nascent adhesion formation with a favorable ratio of nascent adhesion turnover vs. myosin-dependent maturation. Here, we address the parallel question of whether or not the more linear, DOCK180-mediated pathway is capable of fostering similar dynamics. This is not so far-fetched, as it might be argued that any integrin-mediated Rac activation pathway will share the core positive feedback structure of protrusion $\rightarrow$ nascent adhesion $\rightarrow$ Rac activation $\rightarrow$ protrusion. Hereafter we refer to this as the core protrusion cycle. In contrast to the $\beta \mathrm{PIX} / \mathrm{Rac} / \mathrm{PAK}$ feedback mechanism, the DOCK180 pathway might not offer the requisite degree of nonlinearity, in which case paxillin phosphorylated on $\mathrm{Tyr}^{31} / \mathrm{Tyr}^{118}$ might offer a different amplification mechanism, in the form of a c-Src/p190RhoGAP pathway that opposes myosin-based contractility $2,3,33$ in a feedforward manner. 


\section{METHODS}

\section{Mathematical Model}

This work explores the characteristics of a mathematical model describing the fundamental coupling of protrusion and adhesion dynamics (Fig. 1). Although it is not spatially resolved, the model includes a coarse-grained representation of the spatial effects important to signaling during cellular protrusion. These spatial effects are included implicitly based on a specific model control volume comprising a small region of a protruding lamella and the relative locations of model components therein. The spatial reference point for the model is the leading edge of a protruding region of the cell, and the modeled control volume represents the region directly behind the protruding edge, encompassing the lamellipodium and a portion of the lamella.

The model equations represent biochemical mechanisms or, where the mechanism is less understood, phenomenological couplings among variables. Nascent adhesions $(n)$ are formed as a consequence of finding new ECM binding sites, a process that depends on protrusion velocity $(v)^{6}$ and the parameter $k_{a, n}^{\mathrm{ECM}}$, which describes the density and character of the ECM. These adhesions either turn over, at a rate that also depends on the protrusion velocity, ${ }^{6}$ or they mature to form stable adhesions. The conservation of $n$ in the control volume is as follows.

$$
\frac{d n}{d t}=k_{a, n}^{\mathrm{ECM}}\left(1+E_{n} v\right)-k_{d, n}\left(1+C_{n} v\right) n-k_{a, s} f(m, x) n .
$$

It is assumed that Rac activity ( $r$; see below) stimulates membrane protrusion in a saturable manner; in dimensionless terms,

$$
v=\frac{K_{v} r}{\left(1+K_{v} r\right) g(s)} .
$$

The functions $f(m, x)$ and $g(s)$ are phenomenological functions, formulated in the simplest way mathematically as follows. $f(m, x)$ characterizes the enhancement of adhesion maturation by myosin ( $m$; see below) and the inhibition of that enhancement by tyrosine-phosphorylated $\left(\mathrm{pTyr}^{31 / 118}\right)$ paxillin $\left(x\right.$; see below). The notion is that $\mathrm{pTyr}^{31 / 118}$-paxillin mediates Srcdependent regulation of Rho.

$$
f(m, x)=1+\frac{E_{s} m}{1+I_{s} x} .
$$

The function $g(s)$ represents a hypothetical inhibition of protrusion by stable adhesions $(s)$, whether by a mechanical or biochemical mechanism, as follows:

$$
g(s)=1+I_{n} s .
$$

Stable adhesions $(s)$ are created through the aforementioned maturation process and then disappear from the control volume either by disassembly or by rearward convection (which depends on protrusion velocity).

$$
\frac{d s}{d t}=k_{a, s} f(m, x) n-k_{d, s}\left(1+C_{s} v\right) s .
$$


Myosin activity is mobilized by stable adhesions, modeled according to the phenomenological equation,

$$
\frac{d m}{d t}=k_{d, m}(s-m) .
$$

$\mathrm{pTyr}^{31 / 118}$-paxillin $(x)$ represents the subset of nascent adhesions associated with paxillin phosphorylated on Y31 and/or Y118, which implicitly represents adhesions associated with DOCK180 and c-Src; phosphorylation/dephosphorylation of paxillin is considered to be fast relative to nascent adhesion formation/turnover/maturation, such that

$$
\frac{d x}{d t} \approx k_{d, x}\left[K_{x}(n-x)-x\right] .
$$

Active Rac $(r)$ is generated in response to $\mathrm{pTyr}^{31 / 118}$-paxillin and the implicit recruitment of DOCK180, modeled as follows:

$$
\frac{d r}{d t}=k_{d, r}(x-r) .
$$

The model parameters were estimated based on experimental evidence where possible, and parameters were systematically varied to determine their effect on protrusion and adhesion dynamics. Initial analysis of the effect of different parameters on system behavior led us to choose the baseline values listed in Table 1 .

Stochastic Simulation of the Model Equations-Stochastic simulations were performed using the Next Reaction Method, ${ }^{11}$ implemented in MATLAB (MathWorks, Natick, MA). The Next Reaction Method is a computational algorithm that simulates trajectories of the chemical master equation describing discrete stochastic systems, such as those encountered in cells where small numbers of reacting species or rare reaction events dominate system dynamics. To relate dimensionless species concentrations (lowercase) to numbers of molecules (uppercase), a number density scaling factor is specified for each state variable. The scaling factor is denoted in uppercase with an asterisk superscript; for example $n=N / N^{*}$. Based on the formulation of Eqs. (1-8), $S^{*}=X^{*}=N^{*}$, and $M^{*}=K_{m} N^{*}$ and $R^{*}=$ $K_{r} N^{*}$, so the additional parameters $N^{*}, K_{m}$, and $K_{r}$ must be specified for stochastic simulations. We assumed $K_{m}=K_{r}=10$, such that the numbers of $R$ and $M$ are somewhat amplified relative to their activators ( $X$ and $S$, respectively), and a scaling factor of $N^{*}=1$ was used.

Stochastic simulation results were characterized by measuring the mean lifetimes of protrusion and adhesion events occurring during a simulation period of $1000 \mathrm{~min}$. Protrusion events were identified as periods of time during which $v>0.5$, and adhesion events were identified as periods of time during which $S \geq 1$.

RESULTS

The Core Protrusion Cycle and Its Inhibition by Stable Adhesions Are Sufficient for Generating the Full Diversity of Protrusion/Adhesion Dynamics at Lower ECM Density, But Not Necessarily at Higher ECM Density-To recapitulate the stochastic dynamics of cell protrusion and adhesion and facilitate comparison of the hypothetical adhesion/protrusion circuit to cellular observations, the hypothetical model was instantiated using a single-compartment stochastic simulation algorithm based on the Next 
Reaction Method. ${ }^{11}$ Of the model parameters (Table 1), four were identified as centrally important in determining diverse protrusion/adhesion phenotypes, ranging from sustained protrusion to sustained adhesion and including periods of transient protrusion and adhesion: those characterizing ECM density $\left(k_{a, n}^{\mathrm{ECM}}\right)$, enhancement of adhesion maturation via myosin contractility $\left(E_{S}\right)$, inhibition of adhesion maturation via Src-dependent signaling $\left(I_{S}\right)$, and inhibition of protrusion by stable adhesions $\left(I_{n}\right)$. Although the crux of the analysis centers on these four, other model parameters were varied systematically to assess their impact on system behavior (Supplementary Figs. S1 and S2). Changes to the parameters affecting formation and turnover of nascent adhesions $\left(E_{n}, C_{n}\right.$, and $\left.k_{d, n}\right)$ tend to shift the maximal protrusion duration to higher or lower values of $k_{a, n}^{\mathrm{ECM}}$, whereas the parameters affecting stable adhesion turnover $\left(C_{s}\right.$ and $k_{d, s}$ ) shift the appearance of longer-lived stable adhesions with respect to myosin contractility $\left(E_{S}\right)$. Alternatively, the saturation constants $K_{v}$ and $K_{x}$ alter the maximal protrusion duration at intermediate $k_{a, n}^{\mathrm{ECM}}$, but do not shift protrusion behavior with respect to $k_{a, n}^{\mathrm{ECM}}$.

A critical function of the signaling network controlling cell migration is the ability to sense and respond to changes in ECM density, a relationship that is influenced by myosin-mediated contractility. ${ }^{12}$ Hence, to quantitatively compare different adhesion/protrusion phenotypes, the mean lifetimes of protrusion and stable adhesion events in stochastic simulations of the model equations were calculated for ranges of values of $k_{a, n}^{\mathrm{ECM}}$ and $E_{S}$ (Figs. $2 \mathrm{a}$ and $2 \mathrm{~b}$, and later in the $a$ and $b$ panels of Figs. 3-6); regions of the parameter space where the system is deterministically bistable (i.e., where it has multiple steady states), identified by numerical analysis, are indicated on the same plots.

To analyze the influence of Src-mediated inhibition of adhesion maturation, we first simulated the core protrusion cycle with no direct inhibition of adhesion maturation $\left(I_{s}=0 ;\right.$ Fig. 2$)$.

Figures $2 \mathrm{c}-2 \mathrm{f}$ show stochastic simulation results for four different parameter sets corresponding to, respectively: low ECM/high myosin, low ECM/low myosin, high ECM/high myosin, and high ECM/low myosin as indicated.

At a lower ECM density (Figs. 2c and 2d), the system exhibits highly protrusive activity, interspersed with pauses. With high myosin feedback (Fig. 2c), the pauses of protrusion activity are usually, but not always, characterized by a switch mediated by formation of stable adhesions (because of the inhibitory mechanism embodied by the parameter $I_{n}$, which is set to a value of 1 here); otherwise, protrusion pauses or dies out because of a stochastically low rate of nascent adhesion formation.

At a higher ECM density (Figs. 2e and 2f), the system exhibits sustained stable adhesions, with only transient protrusive activity following initiation of the simulation. Because simulations are initiated with all state variables at zero, this observation indicates that with no bias in statespace, the system tends to evolve toward stable adhesion and not protrusion under these conditions. As the ECM density parameter is increased, the model behavior transitions from dynamic protrusion $\left(k_{a, n}^{\mathrm{ECM}}=0.1 \mathrm{~min}^{-}\right)$to stable adhesion $\left(k_{a, n}^{\mathrm{ECM}}=1 \mathrm{~min}^{-}\right)$via incremental decreases in the number and duration of protrusion events (analysis not shown).

\section{Complementing the Core Protrusion Cycle with Feedforward Inhibition of Adhesion Maturation Facilitates Transient Protrusion at High ECM Density by Increasing the Frequency of Protrusion Events-Although the signaling network} lacking Src-mediated, feedforward inhibition of adhesion maturation is capable of generating transient adhesion and protrusion behavior at low ECM, it does not support stochastically transient protrusion at high ECM. Addition of Src-mediated inhibition of adhesion maturation, with $I_{S}=1$ (Fig. 3), facilitates transient protrusion at the higher ECM density (Fig. 3f). The 
observation that Src-mediated, feedforward regulation supports transient protrusions at high ECM suggests that opposing mechanisms that encourage adhesion formation and maturation, respectively, might be necessary to represent adhesion and protrusion dynamics accurately. High myosin activity coupled with high ECM, however, results in sustained stable adhesions that prevent protrusion even with moderate levels of Src-mediated inhibition (Fig. 3e).

\section{Interplay Between Src-Mediated Inhibition of Adhesion Maturation and Adhesion-Mediated Inhibition of Protrusion Controls Protrusion/Adhesion Dynamics at High ECM Density-Increasing the effect of Src-mediated feedforward} regulation, as by setting $I_{S}=10$ (Fig. 4), produces little change in protrusion/adhesion dynamics at the lower ECM density, except for an apparent inability of the transient switching to adhesion stabilization to take hold (Fig. 4c). The more striking change is seen at the higher ECM density; increasing the influence of the Src-mediated feedforward mechanism counteracts the effects of high myosin contractility, supporting transient protrusion behavior (compare Fig. 4e with Fig. 3e).

Stronger inhibition of protrusion by stable adhesions, implemented by increasing the value of $I_{n}$ to 10 (with $I_{s}=1$ ), antagonizes the Src-mediated effect described above (Fig. 5). As expected, this adjustment yields more transient protrusions at lower ECM (Fig. 5c) and prevents transient protrusions at higher ECM and high myosin (Fig. 5e).

From there, a proportional increase in the Src-mediated inhibition of adhesion maturation $\left(I_{S}\right.$ $=I_{n}=10$; Fig. 6 ) is not sufficient to counteract the protrusion inhibition at high ECM and myosin (Fig. 6e); indeed, the behavior is similar to the case where both regulatory mechanisms are less active (compare to Fig. 3, with $I_{s}=I_{n}=1$ ), indicating that the balance of the two regulatory mechanisms is what determines the qualitative behavior.

Consistent with this notion, increasing the value of $I_{S}$ such that protrusion is once again favored $\left(I_{s}=100, I_{n}=10\right)$ permits transient protrusion even under high ECM/high myosin conditions (Fig. 7). The Src-mediated regulation of adhesion maturation apparently balances the effect of protrusion inhibition to produce periods of both sustained adhesion and sustained protrusion, provided that this "buffering" mechanism is strong enough. Moreover, the frequency of transient protrusion is tuned in the model by co-modulating the values of $I_{s}$ and $I_{n}$ (compare Fig. 7 with Fig. 4e, with $I_{s}=10, I_{n}=1$ ).

\section{DISCUSSION}

Two Rac Activation Pathways: Similar Dynamics Achieved by Different Mechanisms-Although several published models have examined the mechanics of cell migration based on broad phenomenological effects, ${ }^{9,27,30}$ the present work examines a more detailed biochemical mechanism for relating adhesion signaling and dynamics to cellular protrusion in lamellipodia. Specifically, we have shown that the DOCK180/Src axis, controlled by tyrosine-phosphorylated paxillin, is sufficient to produce transient periods of adhesion and protrusion over a range of ECM densities and myosin activity levels.

Our parallel analysis of the bPIX/Rac/PAK pathway suggests that amplification of the core protrusion cycle by the PAK $\rightarrow$ paxillin feedback mechanism can produce transient adhesion and protrusion dynamics (Cirit et al., submitted), and the current study shows how the DOCK180/Src axis produces similar adhesion/protrusion dynamics via a feedforward "buffering of inhibition" mechanism. Thus, it appears that the governance of adhesion/ protrusion dynamics may be achieved by different signaling mechanisms. Instead of amplifying nascent adhesion-mediated signaling directly, the $\mathrm{Src} \rightarrow$ RhoGAP mechanism promotes protrusion by inhibiting the competing process of adhesion maturation; these protrusions are rendered transient once maturation over-whelms the "buffer." 
Switching Between DOCK180/Rac-Mediated Protrusion and Myosin-Mediated Adhesion Maturation Depends on the Balanced Interplay of Negative Regulation Mechanisms-Consistent with the buffering concept, our modeling results indicate that, if the core protrusion cycle is to generate metastable protrusion behavior, the effects of protrusion inhibition by stable adhesions and Src-mediated inhibition of adhesion maturation must be properly balanced. Simulation results show that the magnitude of the Src-mediated feedforward mechanism must be somewhat greater than that of the protrusion inhibition mechanism to facilitate protrusion at high ECM and myosin; however, high Src-mediated inhibition of adhesion maturation does not prevent adhesion formation at low ECM and/or low myosin. In that sense, the Src-mediated buffering of adhesion maturation renders the system more robust to different ECM and myosin conditions while facilitating transient protrusion and adhesion. Such a signaling mechanism presents an attractive means for maintaining sensitivity to changes in ECM density or myosin activity across wide ranges of these variables.

Switching Behavior in the Model is not Associated with Bistability-Intuitively, it is tempting to propose that regions of parameter space that support bistability would create opportunities for a stochastic system to switch between steady states; this, however, is not the case for the present model. The behavior in or near regions of bistability is such that stochastic trajectories exclusively track toward the stable adhesion state. In fact, parameter sets for which the size of the bistable region was diminished were more effective in generating frequent switching between protrusion and adhesion maturation.

Implications for Control of Cell Motility: Putting the Model to the Test-The ability of cells to spread and migrate on a broad range of ECM concentrations and under the regulatory control of actomyosin appears to rely upon signaling circuits with balanced feedback and feedforward interactions that facilitate sensitivity and adaptability to different ECM conditions. Our analysis of one such circuit leads to a number of hypotheses/predictions regarding the influence of Src/RhoGAP-mediated signaling on protrusion and adhesion dynamics at the cell's leading edge(s). Diminishing the ability of this pathway to inhibit adhesion maturation is expected to impede protrusion at higher-than-optimal ECM density, whereas this mechanism is predicted to have less of an effect at lower ECM density. One should be able to titrate the magnitude of this perturbation by manipulating myosin levels; i.e., the effect should be tempered by partial knockdown of myosin IIA. Likewise, amplification of the Src-mediated inhibition mechanism should be able to counteract the maturation-promoting effect of myosin overexpression. Of course, these are not trivial experiments, in no small part because Src-family kinases have many functions in addition to the mechanism considered in the model.

A deficiency in the proposed signaling circuit is also expected to affect the directional persistence of migration. ${ }^{24}$ Previous work has suggested that chemotaxis involves a subtle bias in the execution of stochastic protrusion events $1,32,35$; if so, the ability of a cell to modulate the frequency of transitions between protrusion and stable adhesion formation is relevant to the fidelity of cell taxis.

\section{Supplementary Material}

Refer to Web version on PubMed Central for supplementary material.

\section{Acknowledgments}

The authors wish to thank Murat Cirit for helpful discussions and guidance on stochastic simulation. This work was supported by the Cell Migration Consortium under National Institutes of Health Grant U54-GM064346. 


\section{ABBREVIATIONS}

$\begin{array}{ll}\text { ECM } & \text { Extracellular matrix } \\ \text { FAK } & \text { Focal adhesion kinase } \\ \text { GEF } & \text { Guanine nucleotide exchange factor }\end{array}$

\section{REFERENCES}

1. Arrieumerlou C, Meyer T. A local coupling model and compass parameter for eukaryotic chemotaxis. Dev. Cell 2005;8:215-227. [PubMed: 15691763]

2. Arthur WT, Burridge K. RhoA inactivation by p190RhoGAP regulates cell spreading and migration by promoting membrane protrusion and polarity. Mol. Biol. Cell 2001;12:2711-2720. [PubMed: 11553710]

3. Arthur WT, Petch LA, Burridge K. Integrin engagement suppresses RhoA activity via a c-Srcdependent mechanism. Curr. Biol 2000;10:719-722. [PubMed: 10873807]

4. Bershadsky AD, Ballestrem C, Carramusa L, Zilberman Y, Gilquin B, Khochbin S, Alexandrova AY, Verkhovsky AB, Shemesh T, Kozlov MM. Assembly and mechanosensory function of focal adhesions: experiments and models. Eur. J. Cell Biol 2006;85:165-173. [PubMed: 16360240]

5. Burridge K, Turner C, Romer L. Tyrosine phosphorylation of paxillin and pp125FAK accompanies cell adhesion to extracellular matrix: a role in cytoskeletal assembly. J. Cell Biol 1992;119:893-903. [PubMed: 1385444]

6. Choi CK, Vicente-Manzanares M, Zareno J, Whitmore LA, Mogilner A, Horwitz AR. Actin and [alpha]-actinin orchestrate the assembly and maturation of nascent adhesions in a myosin II motorindependent manner. Nat. Cell Biol 2008;10:1039-1050. [PubMed: 19160484]

7. Cirit M, Krajcovic M, Choi CK, Welf ES, Horwitz AF, Haugh JM. Stochastic model of integrinmediated signaling and adhesion dynamics at the leading edges of migrating cells. PLoS Comput. Biol 2010;6:e1000688. [PubMed: 20195494]

8. Deshpande VS, McMeeking RM, Evans AG. A bio-chemo-mechanical model for cell contractility. Proc. Natl. Acad. Sci. USA 2006;103:14015-14020. [PubMed: 16959880]

9. DiMilla PA, Barbee K, Lauffenburger DA. Mathematical model for the effects of adhesion and mechanics on cell migration speed. Biophys. J 1991;60:15-37. [PubMed: 1883934]

10. Galbraith CG, Yamada KM, Galbraith JA. Polymerizing actin fibers position integrins primed to probe for adhesion sites. Science 2007;315:992-995. [PubMed: 17303755]

11. Gibson M, Bruck J. Efficient exact stochastic simulation of chemical systems with many species and many channels. J. Phys. Chem. A 2000;104:1876-1889.

12. Gupton SL, Waterman-Storer CM. Spatiotemporal feedback between actomyosin and focal-adhesion systems optimizes rapid cell migration. Cell 2006;125:1361-1374. [PubMed: 16814721]

13. Jaffe A, Hall A. Rho GTPases: biochemistry and biology. Ann. Rev. Cell Dev. Biol 2005;21:247269. [PubMed: 16212495]

14. Jones NP, Katan M. Role of phospholipase Cgamma1 in cell spreading requires association with a beta-Pix/GIT1-containing complex, leading to activation of Cdc42 and Rac1. Mol. Cell. Biol 2007;27:5790-5805. [PubMed: 17562871]

15. Kiyokawa E, Matsuda M. Regulation of focal adhesion and cell migration by ANKRD28-DOCK180 interaction. Cell Adh. Migr 2009;3:281-284. [PubMed: 19458477]

16. Lee J, Jung ID, Chang WK, Park CG, Cho DY, Shin E, Seo DW, Kim YK, Lee HW, Han J, et al. p85 beta-PIX is required for cell motility through phosphorylations of focal adhesion kinase and p38 MAP kinase. Exp. Cell Res 2005;307:315-328. [PubMed: 15893751]

17. Lock JG, Wehrle-Haller B, Strömblad S. Cell-matrix adhesion complexes: master control machinery of cell migration. Semin. Cancer Biol 2008;18:65-76. [PubMed: 18023204]

18. Mogilner A. Mathematics of cell motility: have we got its number? J. Math. Biol 2009;58:105-134. [PubMed: 18461331] 
19. Moissoglu K, Slepchenko BM, Meller N, Horwitz AF, Schwartz MA. In vivo dynamics of Racmembrane interactions. Mol. Biol. Cell 2006;17:2770-2779. [PubMed: 16597700]

20. Nayal A, Webb DJ, Brown CM, Schaefer EM, Vicente-Manzanares M, Horwitz AR. Paxillin phosphorylation at Ser273 localizes a GIT1-PIX-PAK complex and regulates adhesion and protrusion dynamics. J. Cell Biol 2006;173:587-589. [PubMed: 16717130]

21. Novak IL, Slepchenko BM, Mogilner A, Loew LM. Cooperativity between cell contractility and adhesion. Phys. Rev. Lett 2004;93:268109. [PubMed: 15698031]

22. Para A, Krischke M, Merlot S, Shen Z, Oberholzer M, Lee S, Briggs S, Firtel RA. Dictyostelium Dock180-related RacGEFs regulate the actin cytoskeleton during cell motility. Mol. Biol. Cell 2009;20:699-707. [PubMed: 19037099]

23. Petit V, Boyer B, Lentz D, Turner CE, Thiery JP, Vallés AM. Phosphorylation of tyrosine residues 31 and 118 on paxillin regulates cell migration through an association with CRK in NBT-II cells. J. Cell Biol 2000;148:957-970. [PubMed: 10704446]

24. Petrie RJ, Doyle AD, Yamada KM. Random versus directionally persistent cell migration. Nat. Rev. Mol. Cell. Biol 2009;10:538-549. [PubMed: 19603038]

25. Ridley AJ, Schwartz MA, Burridge K, Firtel RA, Ginsberg MH, Borisy G, Parsons JT, Horwitz AR. Cell migration: integrating signals from front to back. Science 2003;302:1704-1709. [PubMed: 14657486]

26. Romer LH, Birukov KG, Garcia JGN. Focal adhesions: paradigm for a signaling nexus. Circ. Res 2006;98:606-616. [PubMed: 16543511]

27. Satulovsky J, Lui R, Wang Y. Exploring the control circuit of cell migration by mathematical modeling. Biophys. J 2008;94:3671-3683. [PubMed: 18199677]

28. Smith HW, Marra P, Marshall CJ. uPAR promotes formation of the p130Cas-Crk complex to activate Rac through DOCK180. J. Cell Biol 2008;182:777-790. [PubMed: 18725541]

29. Steffen A, Rottner K, Ehinger J, Innocenti M, Scita G, Wehland J, Stradal TEB. Sra-1 and Nap1 link Rac to actin assembly driving lamellipodia formation. EMBO J 2004;23:749-759. [PubMed: 14765121]

30. Stéphanou A, Mylona E, Chaplain M, Tracqui P. A computational model of cell migration coupling the growth of focal adhesions with oscillatory cell protrusions. J. Theor. Biol 2008;253:701-716. [PubMed: 18550085]

31. ten Klooster JP, Jaffer ZM, Chernoff J, Hordijk PL. Targeting and activation of Rac1 are mediated by the exchange factor beta-Pix. J. Cell Biol 2006;172:759-769. [PubMed: 16492808]

32. Tranquillo RT, Lauffenburger DA. Stochastic model of leukocyte chemosensory movement. J. Math. Biol 1987;25:229-262. [PubMed: 3625051]

33. Tsubouchi A, Sakakura J, Yagi R, Mazaki Y, Schaefer E, Yano H, Sabe H. Localized suppression of RhoA activity by Tyr31/118-phosphorylated paxillin in cell adhesion and migration. J. Cell Biol 2002;159:673-683. [PubMed: 12446743]

34. Vallés AM, Beuvin M, Boyer B. Activation of Rac1 by paxillin-Crk-DOCK180 signaling complex is antagonized by Rap1 in migrating NBT-II cells. J. Biol. Chem 2004;279:44490-44496. [PubMed: 15308668]

35. van Haastert PJM, Postma M. Biased random walk by stochastic fluctuations of chemoattractantreceptor interactions at the lower limit of detection. Biophys. J 2007;93:1787-1796. [PubMed: 17513372]

36. Vogel V, Sheetz M. Cell fate regulation by coupling mechanical cycles to biochemical signaling pathways. Curr. Opin. Cell Biol 2009;21:38-46. [PubMed: 19217273]

37. Volinsky N, Gantman A, Yablonski D. A Pak- and Pix-dependent branch of the SDF-1alpha signalling pathway mediates T cell chemotaxis across restrictive barriers. Biochem. J 2006;397:213-222. [PubMed: 16515536]

38. Webb DJ, Donais K, Whitmore LA, Thomas SM, Turner CE, Parsons JT, Horwitz AF. FAK-Src signalling through paxillin, ERK and MLCK regulates adhesion disassembly. Nat. Cell Biol 2004;6:154-161. [PubMed: 14743221]

39. Webb DJ, Kovalenko M, Whitmore L, Horwitz AF. Phosphorylation of serine 709 in GIT1 regulates protrusive activity in cells. Biochem. Biophys. Res. Comm 2006;346:1284-1288. [PubMed: 16797488] 
40. Zaidel-Bar R, Cohen M, Addadi L, Geiger B. Hierarchical assembly of cell-matrix adhesion complexes. Biochem. Soc. Trans 2004;32:416-420. [PubMed: 15157150]

41. Zamir E, Geiger B. Molecular complexity and dynamics of cell-matrix adhesions. J. Cell Sci 2001;114:3583-3590. [PubMed: 11707510]

42. Zhao ZS, Manser E, Loo TH, Lim L. Coupling of PAK-interacting exchange factor PIX to GIT1 promotes focal complex disassembly. Mol. Cell. Biol 2000;20:6354-6363. [PubMed: 10938112] 


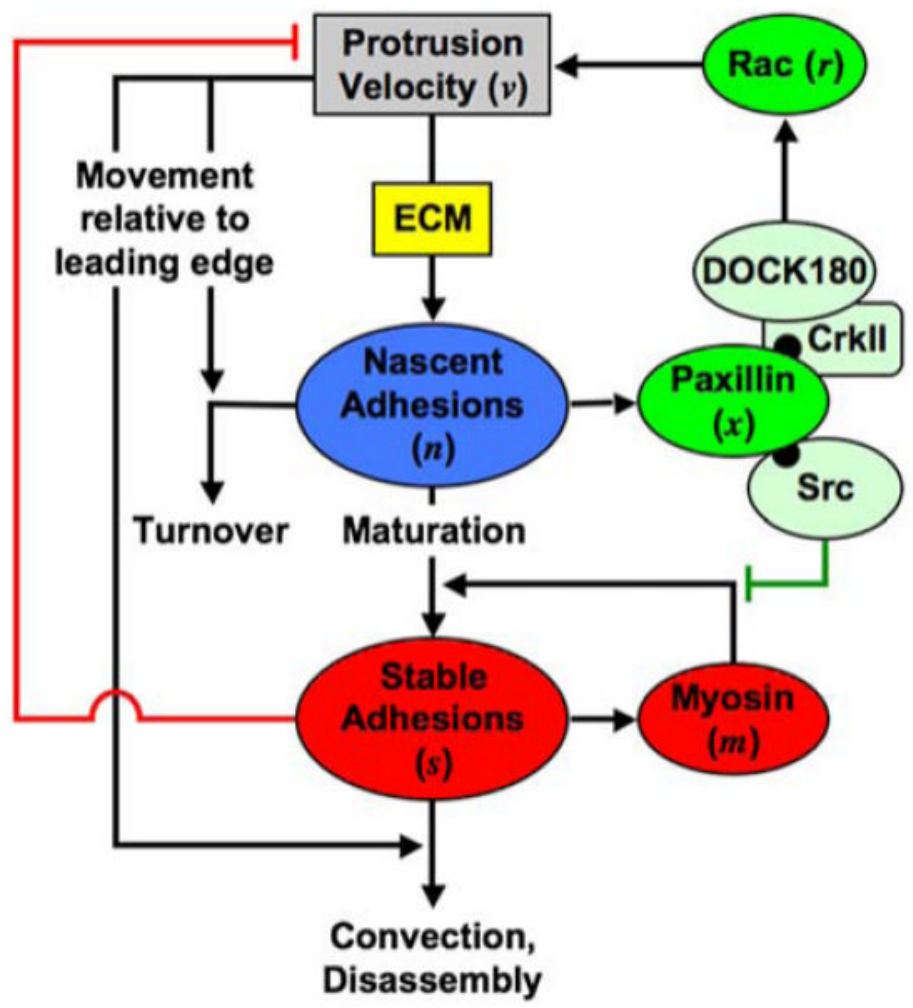

FIGURE 1.

Conceptual model framework. 

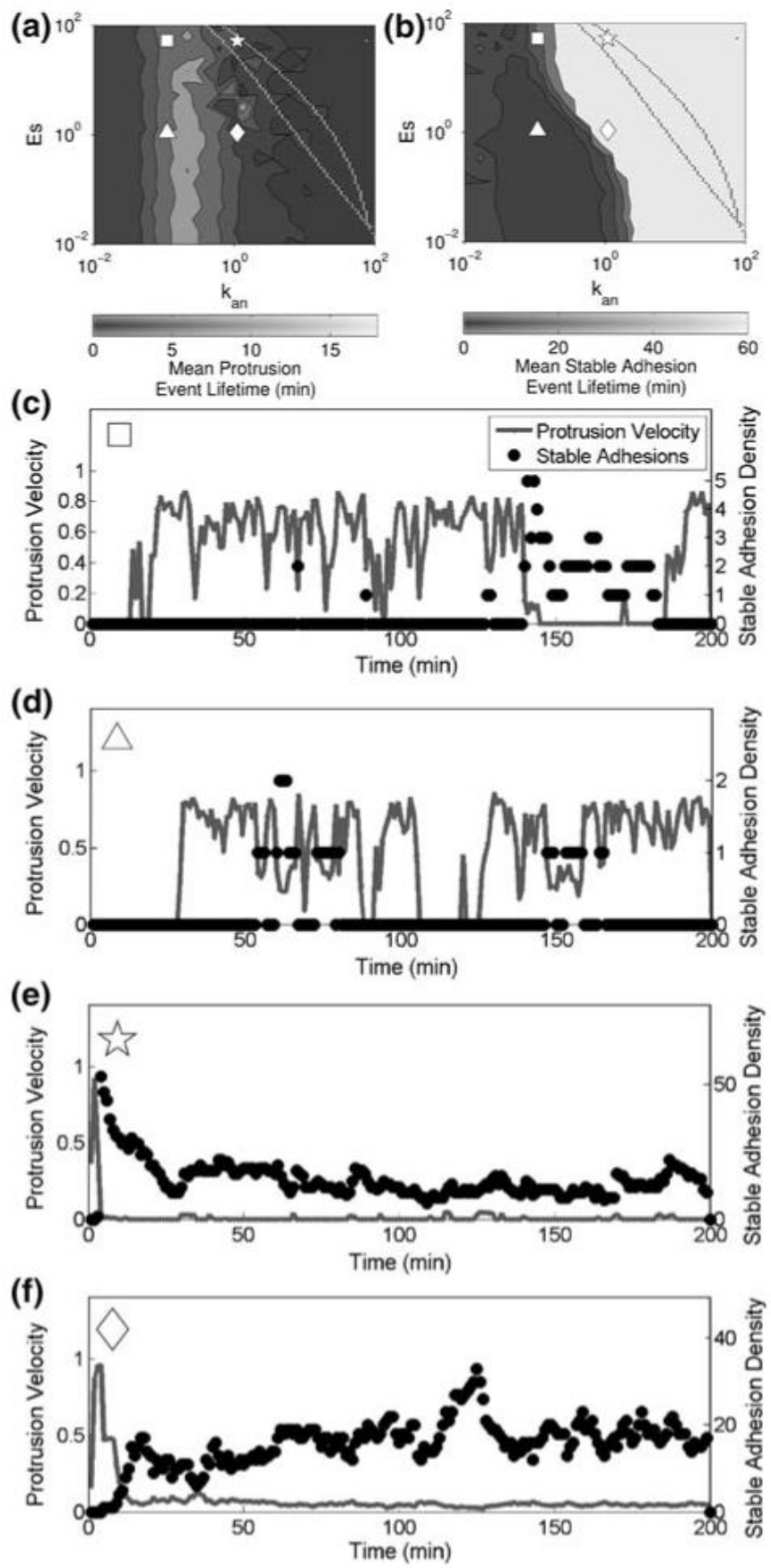

FIGURE 2.

Stochastic simulation of protrusion/adhesion dynamics with low protrusion inhibition and no Src-mediated inhibition of adhesion maturation. $(a, b)$ Mean lifetimes of protrusion (a) and stable adhesion events (b) are calculated as described in "Methods" section from stochastic simulation results performed over $t=[0,1000]$, with $I_{n}=1$ and $I_{s}=0$. (c-f) Example simulation results corresponding to the parameter values given by the symbols in (a) and (b): (c) $k_{a, n}^{\mathrm{ECM}}=0.1 \mathrm{~min}^{-}, E_{s}=50$; (d) $k_{a, n}^{\mathrm{ECM}}=0.1 \mathrm{~min}^{-}, E_{s}=1$; (e) $k_{a, n}^{\mathrm{ECM}}=1 \min ^{-}, E_{s}=50 ;(f) k_{a, n}^{\mathrm{ECM}}=1$ $\min ^{-}, E_{s}=1$. 

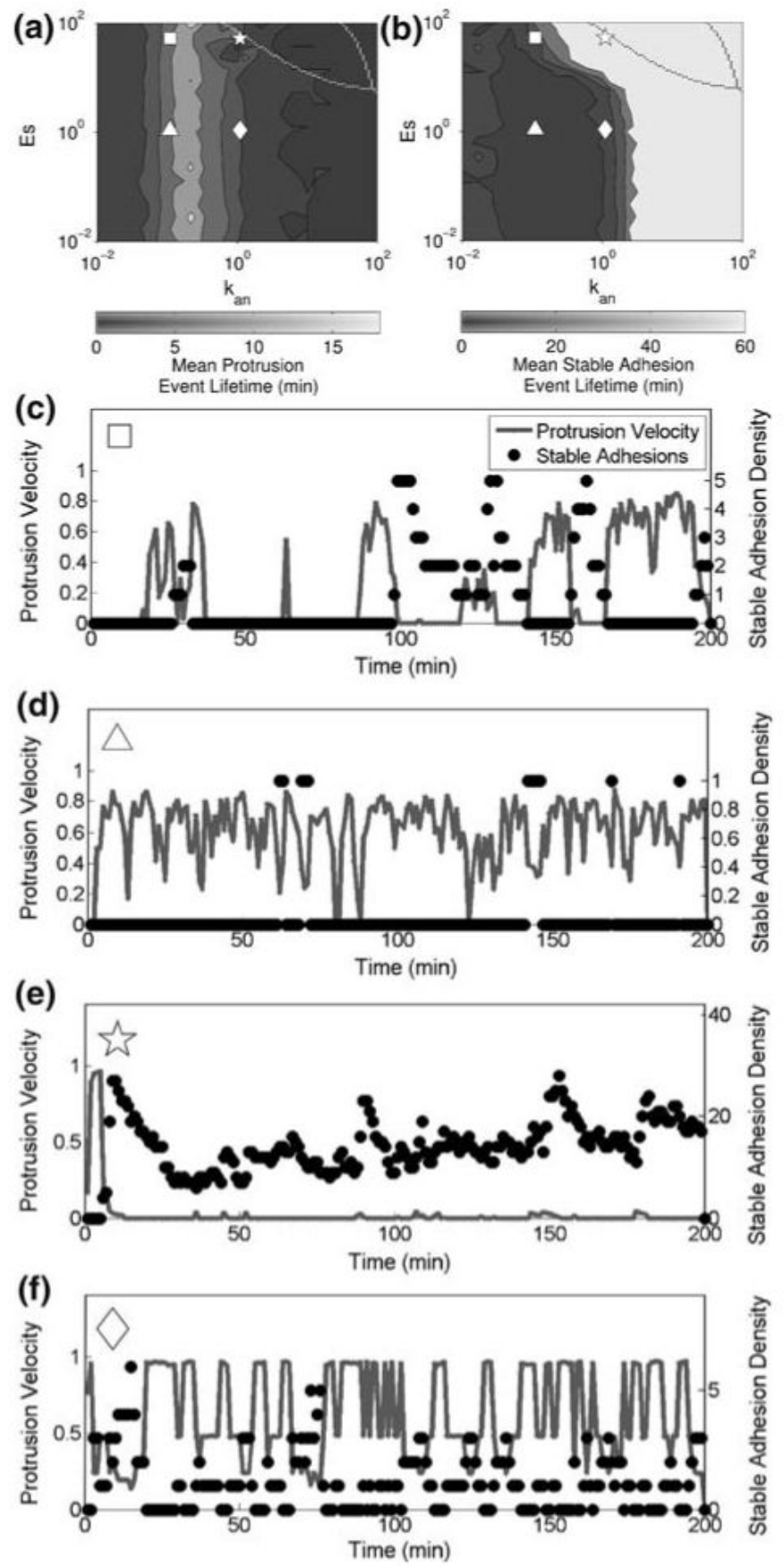

FIGURE 3.

Stochastic simulation of protrusion/adhesion dynamics with low protrusion inhibition and low Src-mediated inhibition of adhesion maturation. $(a, b)$ Mean lifetimes of protrusion (a) and stable adhesion events (b) are calculated as described in "Methods" section from stochastic simulation results performed over $t=[0,1000]$, with $I_{n}=I_{s}=1$. (c-f) Example simulation results corresponding to the parameter values given by the symbols in (a) and (b): (c) $k_{a, n}^{\mathrm{ECM}}=0.1 \mathrm{~min}^{-}, E_{s}=50$; (d) $k_{a, n}^{\mathrm{ECM}}=0.1 \mathrm{~min}^{-}, E_{s}=1$; (e) $k_{a, n}^{\mathrm{ECM}}=1 \mathrm{~min}^{-}, E_{s}=50 ;(f) k_{a, n}^{\mathrm{ECM}}=1$ $\min ^{-}, E_{s}=1$. 

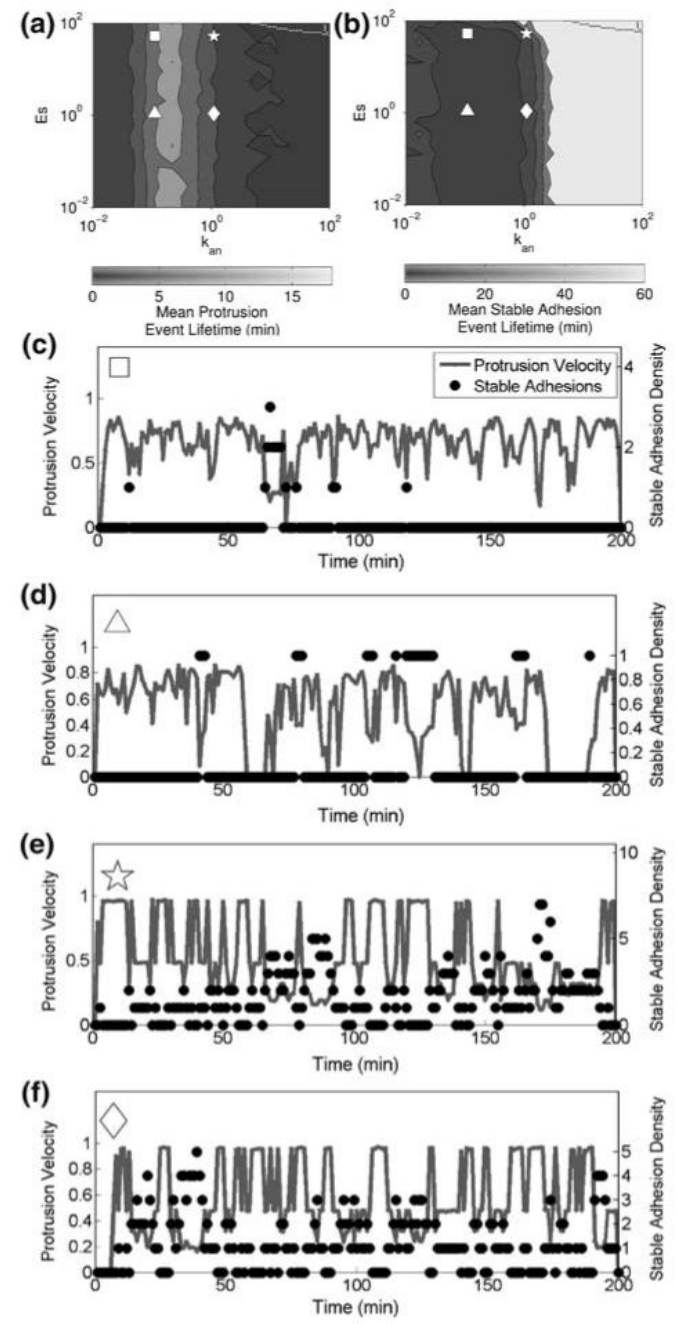

FIGURE 4.

Stochastic simulation of protrusion/adhesion dynamics with low protrusion inhibition and high Src-mediated inhibition of adhesion maturation. $(a, b)$ Mean lifetimes of protrusion (a) and stable adhesion events (b) are calculated as described in "Methods" section from stochastic simulation results performed over $t=[0,1000]$, with $I_{n}=1$ and $I_{S}=10$. (c-f) Example simulation results corresponding to the parameter values given by the symbols in (a) and (b): (c)

$k_{a, n}^{\mathrm{ECM}}=0.1 \mathrm{~min}^{-}, E_{s}=50$; (d) $k_{a, n}^{\mathrm{ECM}}=0.1 \mathrm{~min}^{-}, E_{s}=1$; (e) $k_{a, n}^{\mathrm{ECM}}=1 \mathrm{~min}^{-}, E_{s}=50$; (f) $k_{a, n}^{\mathrm{ECM}}=1$ $\min ^{-}, E_{s}=1$. 

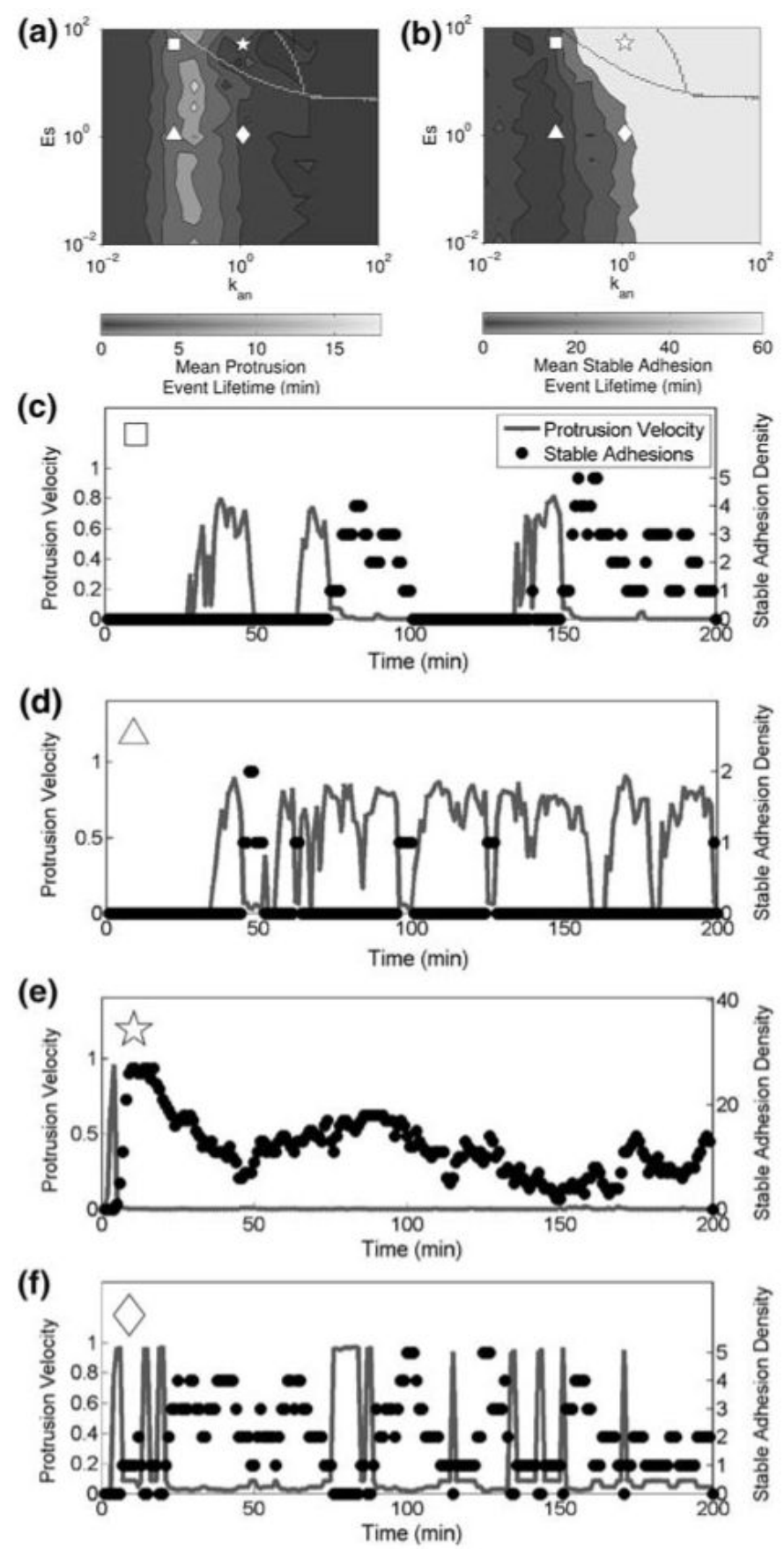

FIGURE 5.

Stochastic simulation of protrusion/adhesion dynamics with high protrusion inhibition and low Src-mediated inhibition of adhesion maturation. (a, b) Mean lifetimes of protrusion (a) and stable adhesion events (b) are calculated as described in "Methods" section from stochastic simulation results performed over $t=[0,1000]$, with $I_{n}=10$ and $I_{s}=1$. (c-f) Example simulation results corresponding to the parameter values given by the symbols in (a) and (b): (c) $k_{a, n}^{\mathrm{ECM}}=0.1 \mathrm{~min}^{-}, E_{s}=50$; (d) $k_{a, n}^{\mathrm{ECM}}=0.1 \mathrm{~min}^{-}, E_{s}=1$; (e) $k_{a, n}^{\mathrm{ECM}}=1 \mathrm{~min}^{-}, E_{s}=50$; (f) $k_{a, n}^{\mathrm{ECM}}=1$ $\min ^{-}, E_{s}=1$. 

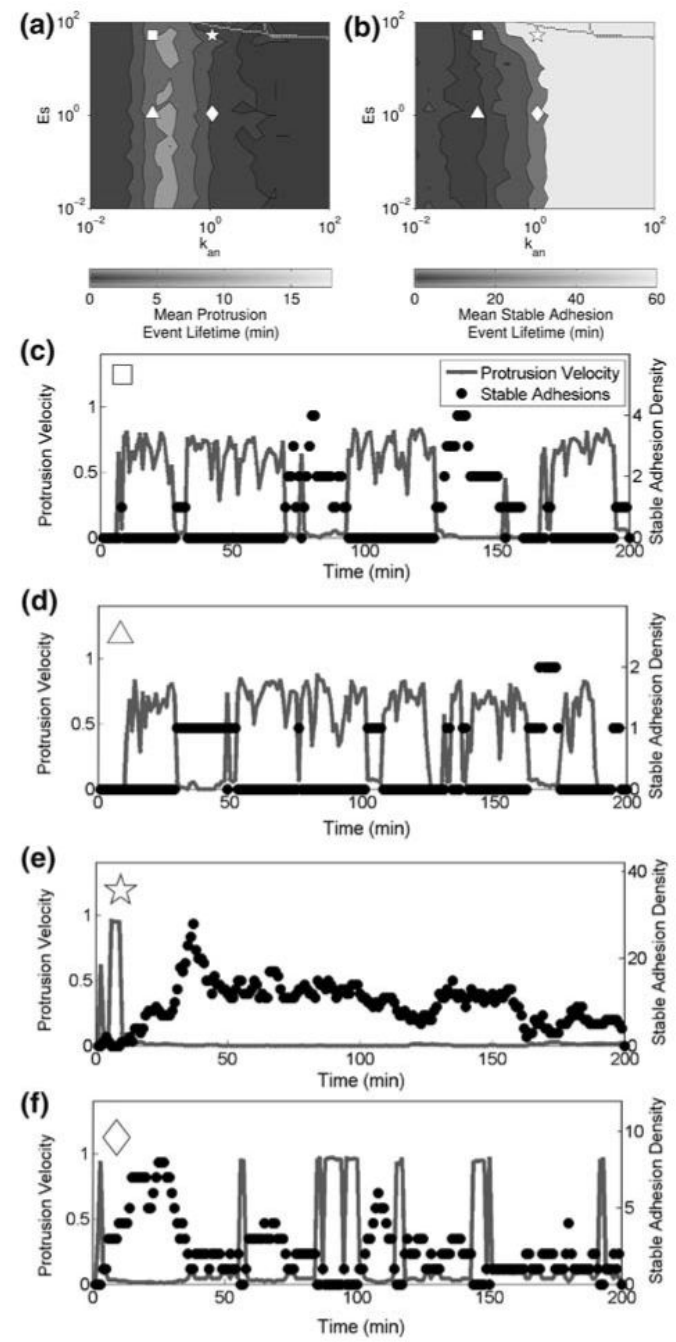

FIGURE 6.

Stochastic simulation of protrusion/adhesion dynamics with high protrusion inhibition and high Src-mediated inhibition of adhesion maturation. ( $a, b)$ Mean lifetimes of protrusion (a) and stable adhesion events (b) are calculated as described in "Methods" section from stochastic simulation results performed over $t=[0,1000]$, with $I_{n}=I_{s}=10$. (c-f) Example simulation results corresponding to the parameter values given by the symbols in (a) and (b): (c)

$k_{a, n}^{\mathrm{ECM}}=0.1 \mathrm{~min}^{-}, E_{s}=50$; (d) $k_{a, n}^{\mathrm{ECM}}=0.1 \mathrm{~min}^{-}, E_{s}=1$; (e) $k_{a, n}^{\mathrm{ECM}}=1 \mathrm{~min}^{-}, E_{s}=50$; (f) $k_{a, n}^{\mathrm{ECM}}=1$ $\min ^{-}, E_{S}=1$. 


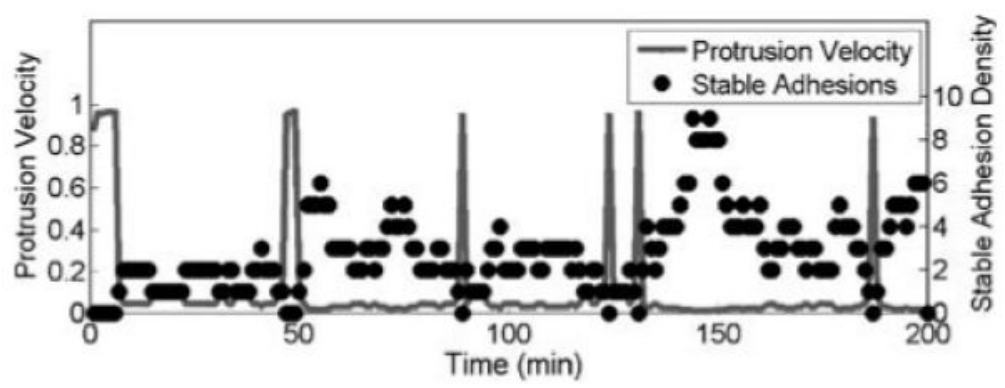

FIGURE 7.

Stochastic simulation of protrusion/adhesion dynamics under conditions corresponding to maturation-promoting conditions (high ECM/high myosin/high protrusion inhibition) and very high Src-mediated inhibition of adhesion maturation. Stochastic simulation results were performed with $k_{a, n}^{\mathrm{ECM}}=1 \mathrm{~min}^{-}, E_{S}=50, I_{n}=10$, and $I_{S}=100$. 
